\title{
Solar-System Studies with Pulsar Timing Arrays
}

\author{
R. N. Caballero ${ }^{1}$ and Collaborators \\ ${ }^{1}$ Max-Planck-Institut für Radioastronomie, Auf dem Hügel 69, 53121 Bonn, Germany \\ email: caball@mpifr-bonn.mpg.de
}

\begin{abstract}
High-precision pulsar timing is central to a wide range of astrophysics and fundamental physics applications. When timing an ensemble of millisecond pulsars in different sky positions, known as a pulsar timing array (PTA), one can search for ultra-low-frequency gravitational waves (GWs) through the spatial correlations that spacetime deformations by passing GWs are predicted to induce on the pulses' times-of-arrival (TOAs). A pulsar-timing model, requires the use of a solar-system ephemeris (SSE) to properly predict the position of the solar-system barycentre, the (quasi-)inertial frame where all TOAs are referred. Here, I discuss how while errors in SSEs can introduce correlations in the TOAs that may interfere with GW searches, one can make use of PTAs to study the solar system. I discuss work done within the context of the European Pulsar Timing Array and the International Pulsar Timing Array collaborations. These include new updates on the masses of planets from PTA data, first limits on masses of the most massive asteroids, and comparisons between SSEs from independent groups. Finally, I discuss a new approach in setting limits on the masses of unknown bodies in the solar system and calculate mass sensitivity curves for PTA data.
\end{abstract}

Keywords. pulsars: general - methods: data analysis, statistical - ephemerides

\section{Introduction}

A pulsar timing array (PTA), is an ensemble of pulsars in various sky positions, and can be employed to search for processes that cause space-correlated signals which will be present in all pulsars (e.g. Foster \& Backer 1990). PTA research is based on precision pulsar timing, i.e. the modelling of pulse times-of-arrival (TOAs), recorded at high precision (e.g. Lorimer \& Kramer 2005). The signals of interest are sought in the timing residuals, i.e. the differences between the observed and model-predicted TOAs, which means that the sensitivity of PTAs to such effects is limited by the rotational stability of the pulsars, which work as celestial clocks. Therefore, PTAs are constructed using the most rotationally stable pulsars known, the millisecond pulsars (MSPs).

PTAs focus primarily on efforts for the direct detection of gravitational waves (GWs) in the frequency regime $10^{-9}-10^{-6} \mathrm{~Hz}$ (e.g. Sesana \& Vecchio 2010). Three collaborations are actively working in the realisation of this goal. Citing their latest data releases, these are the European Pulsar Timing Array (EPTA; Desvignes et al. 2016) in Europe, the Parkes Pulsar Timing Array (PPTA; Reardon et al. 2016) in Australia, and the NorthAmerican Nanohertz Observatory for Gravitational Waves (NANOGrav; Arzoumanian et al. 2015) in North America. These collaborations work together under the International Pulsar Timing Array consortium (IPTA; Verbiest et al. 2016), in an effort to improve both the sensitivity and the robustness of the data and data analyses.

PTA sensitivity to GWs is particularly limited by possible spatially-correlated signals other than GWs (e.g. Tiburzi et al. 2016), particularly in the lower-to-intermediate signal-to-noise regime. The two most frequently studied sources of spatially-correlated 
noise are possible errors in the terrestrial time-standards to which all TOAs are referred and in the solar-system ephemeris (SSE) used. While PTAs are actively working into mitigating these sources of noise, their understanding actually allows PTAs to simultaneously expand their scientific studies. In relation with these noise components specifically, PTA data are used to construct a pulsar-based time-scale of such long-term stability that it can serve as an independent cross-check for the highly precise time-scales from atomic clocks Hobbs et al. (2012), and to provide constraints on possible errors in SSEs and the masses of solar-system planets (Champion et al. 2010, henceforth CHM10). Here, we focus on the latter application and discuss work conducted within the framework of the regional PTAs and the IPTA.

\section{Solar-system ephemerides and Pulsar Timing}

One of the basic reasons that pulsar-timing models use SSEs, is to predict the position of the solar-system barycentre (SSB) for every observing epoch. This is essential, since before calculating the pulse-emission time at the pulsar's co-moving frame, we refer all TOAs to the SSB, a common (quasi-)inertial reference frame. Using PPTA data, CHM10 were the first to use pulsar timing to constrain the masses of planets. The developed method was focused on the approximation of small errors in the planetary masses used by the SSE, i.e. when the error is much smaller than the total mass of the planet. In such an approximation, the effect of the small error in mass, $\delta m$, is a small displacement of the SSB along barycentric position vector of the planet, with respect to the original SSB position. One can show how this delay depends on the pulsar position, by expressing the timing signature of such an effect in terms of the differences in the ecliptic latitude and longitude between the (i-th) planet and the (j-th) pulsar, $\Delta \beta_{i, j}$ and $\Delta \lambda_{i, j}$ respectively, as

$$
\tau_{b} \propto|\boldsymbol{b}| \delta m_{i} \cos \left(\Delta \beta_{i, j}\right) \cos \left(\Delta \lambda_{i, j}\right)
$$

where $|\boldsymbol{b}|$ is the determinant of the barycentric position vector of the planet. It is apparent, that fitting multiple pulsars located in as many different sky position as possible with $\delta m$ as a global fit parameter, improves the quality of the $\delta m$ estimation.

Using four MSPs, CHM10 found that the possible errors in the masses where consistent with zero at the $2 \sigma$ level. The data length of the pulsars ranged from 5.2 to $22.1 \mathrm{yr}$, which meant that for the giant planets with longer periods, the measurements should improve significantly once more MSPs contribute with time-spans longer than the orbital period of Jupiter (11.86 yr). Finally, CHM10 used only one specific SSE. Knowing that there are differences between SSEs, as discussed below, an interesting idea has been to see whether such differences will affect the measured planetary masses by PTAs.

\section{Current and future work}

Various groups are creating SSEs, primarily used for space-mission navigation. In return, in situ measurements of planetary masses by spacecrafts such as the Pioneer and the Voyager provide input for future SSEs. So far, pulsar timing have primarily been using SSEs from two independent groups, namely the DE and the INPOP series of SSEs from the Jet Propulsion Laboratory (JPL) and the Institut de Mécanique Céleste et de Calcul des Éphémérides (IMCCE), respectively. These ephemerides are created using a wealth of data from sources such as optical astrometry, spacecraft mass measurements, radar and laser ranging, etc (see CHM10 and references therein), which are used as input in numerical integrations of the planetary equations of motion. 

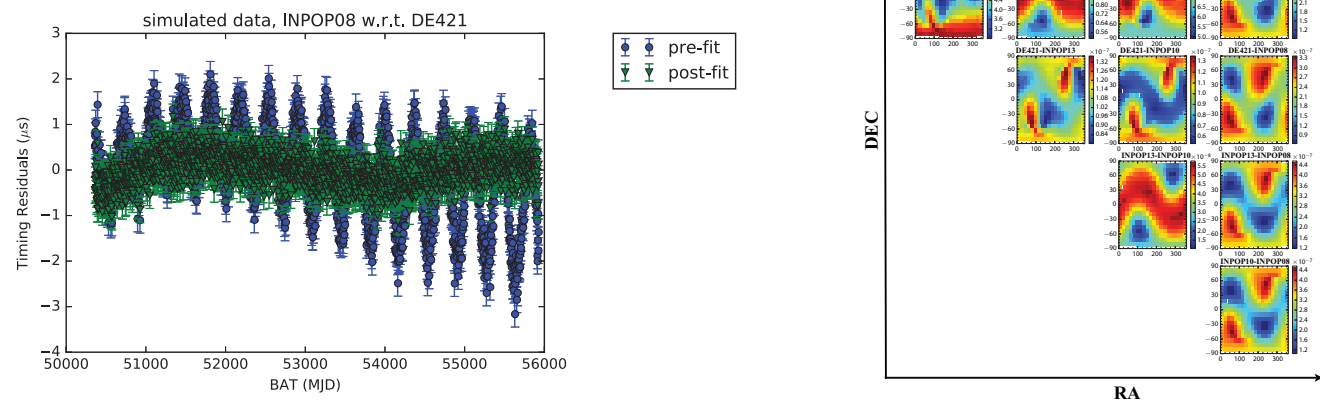

Figure 1. Left Panel: Timing residuals of the difference between the SSEs DE421 and INPOP08, based on simulated data. The figure emphasises the effects of fitting for the pulsar timing model. The blue circles correspond to the difference before fitting the pulsar modes (pre-fit) and have a larger root-mean-square than the residuals after fitting the pulsar model (post-fit), denoted with the green triangles. Right Panel: Difference in the timing residuals for various pairs of SSEs, as a function of sky position, expressed in Right Ascension (RA) and Declination (DEC). The colour-code (for colour prints) is the root-mean-square of the residuals. For each case, we used simulations of 400 MSPs isotropically distributed in the sky. Note how for some pairs the difference is stronger along the ecliptic plane, while for other pairs, away from it.

JPL and IMCCE regularly present differences between the various published SSEs. In pulsar timing, it is important to note that the timing-model fit will absorb some of these differences, biasing some of the parameters. For example, annual, linear and quadratic variations are absorbed by fitting of the pulsar position, pulsar rotational spin and spindown, respectively. In Fig. 1 (left panel), we see such a difference for simulated data of PSR J1713+0747, with TOA uncertainties reflecting those in the published EPTA data. To compare the SSEs, we simulate data using one SSE and then fit the timing model using another SSE. In order to understand the effects of using different SSEs in EPTA work, we examined the residuals of the difference between SSEs in many sky positions. Fig. 1 (right panel) shows the the root-mean-square of the post-fit timing residuals for the difference of various pairs of SSEs. For each case, we used 400 MSPs isotropically distributed in the sky. In order to make the effects prominent we simulated the data with a precision of only a few ns. One can see, that as our signal-to-noise improves, if PTAs are limited by the number of MSPs contributing to the measurement of correlated effects, using different SSEs will affect our sensitivity to the errors in planet masses.

Within the context of the IPTA, we are using data from the first official data release Verbiest et al. (2016) to extend on the work of CHM10. The IPTA data set constitutes a significant improvement thanks to increased data-span and timing precision for multiple of the most rotationally stable MSPs, better observing-frequency coverage, increased number of MSPs contributing to the solutions, and more sophisticated methods for modelling the noise of individual pulsars (e.g. Lentati et al. 2016). Proper noise models are of central importance. Indeed, CHM10 excluded their most precisely-timed MSP from the mass measurement of Mars due to insufficiencies in the noise model. Preliminary results with the IPTA, indicate improvements of factors $4-10$ by comparison to CHM10, depending on the planet. By comparison to only using EPTA data, the IPTA data set, which combines data from all three PTAs, gives an additional improvement of up to a factor $\sim 4$. Furthermore, the IPTA data are now sensitive to mass errors in large objects in the asteroid belt, with initial results constraining the mass of Ceres with precision only an order of magnitude below that published by the IAU Luzum et al. (2011). Our 
IPTA work also repeats the analysis for multiple SSEs from both the JPL and IMCCE. Initial results that compare DE421, DE430, DE435 and INPOP06C showed results that are statistically consistent. Unsurprisingly, deviations start becoming more prominent for the giant planets. This is due to the limited number of orbits completed within the data time-span (if any) and to the higher degree of correlation between the timing signals and the pulsar's low-frequency noise components, and the rotational spin period and period derivative.

As our data become more sensitive, errors in masses that we may measure for the giant planets could be due to errors in the mass determination of their satellites, since PTAs are in fact sensitive to the planetary systems, rather than the planets or their satellites individually. For example, in the case of DE421, five Jovian and nine Saturnian satellites were used in the model (CHM10). As such, dynamical modelling of solar-system bodies in PTAs may allow to shed light in the origin of possible mass-error measurements. As a first step, we use a dynamical model to calculate the effects on the pulsar residuals from unknown bodies in Keplerian orbits around the SSB, essentially accounting only for the gravitational effects of the Sun (Guo, Lee \& Caballero; submitted), and estimate the IPTA-data sensitivity to such objects. We note that this analysis is valid for any type of object, even hypothetical, such as dark-matter clumps Loeb \& Zaldarriaga (1997). With increased precision, time-span and number of pulsars, it is possible that the IPTA will eventually become sensitive to masses of the order of official IAU uncertainties for the Jovian system. In the future, we would focus on expanding the dynamical model to include higher-order effects, such as perturbations from objects other than the Sun, allowing PTAs to also study the effects of satellites in orbit with giant planets and contribute more significantly to the development of SSEs. Our results on constraining the masses of planets and unknown bodies in the solar system using the IPTA will be published in an IPTA paper (Caballero et al. IPTA Collaboration; in prep.).

\section{Acknowledgments}

The ongoing work discussed here is conducted in the framework of the EPTA and the IPTA, and all members who contributed through instrument development, observations, data analysis and algorithm development are acknowledged. Results discussed in this contribution are based on data collected with Effelsberg Radio Telescope, the Nançay Radio Telescope, the Lovell Telescope, the Westerbork Radio Synthesis Telescope, the Parkes Radio Telescope, the Green Bank Telescope, and the Arecibo Radio Telescope.

\section{References}

Arzoumanian, Z. et al. NANOGrav Collaboration 2015, ApJ, 813, 65

Champion, D. J., Hobbs, G. B., \& Manchester, R. N. et al. 1997, ApJ, 720, L201

Desvignes, G., Caballero, R. N., \& Lentati, L. et al. 2016, MNRAS, 458, 3341

Foster, R. S. \& Backer, D. C. 1990, ApJ, 361, 300

Hobbs, G. \& Coles, W., Manchester R. N. et al. 2012, MNRAS, 427, 2780

Lentati L., Shannon, R. M. \& Coles, W. A. 2016, MNRAS, 458, 2161

Loeb, A. \& Zaldarriaga, M. 1997, Phys. Rev. D, 71, 103520

Lorimer, D. R. \& Kramer, M. 2005, Handbook of Pulsar Astronomy. Cambridge Univ. Press

Luzum, B., Capitaine, N., \& Fienga, A. et al. 2011, Cel. Mech. E Dynamical Astron., 110, 293,

Reardon, D. J., Hobbs, G., \& Coles, W. et al. 2016, MNRAS, 455, 1751

Sesana, A. \& Vecchio, A. 2010, Phys. Rev. D, 81, 104008

Tiburzi, C. \& Hobbs, G., Kerr 2016, MNRAS, 455, 4339

Verbiest, J. P. W., Lentati, L., \& Hobbs, G. et al. 2016, MNRAS, 458, 1267 